\title{
Using Huygens Multipole Arrays to Realize Unidirectional Needle-Like Radiation
}

\author{
Richard W. Ziolkowski" \\ University of Technology Sydney, Ultimo NSW 2007, Australia \\ The University of Arizona, Tucson, Arizona 85721, USA \\ (Received 19 December 2016; published 26 July 2017)
}

\begin{abstract}
For nearly a century, the concept of needle radiation has captured the attention of the electromagnetics communities in both physics and engineering, with various types of contributions reoccurring every decade. With the near-term needs for highly directive, electrically small radiators and scatterers for a variety of communications and sensor applications, superdirectivity has again become a topic of interest. While it is well known that superdirective solutions exist and suffer ill-posedness issues in principle, a detailed needle solution has not been reported previously. We demonstrate explicitly, for the first time, how needle radiation can be obtained theoretically from currents driven on an arbitrary spherical surface, and we explain why such a result can only be attained in practice with electrically large spheres. On the other hand, we also demonstrate, more practically, how broadside radiating Huygens source multipoles can be combined into an end-fire array configuration to achieve needle-like radiation performance without suffering the traditional problems that have previously plagued superdirectivity.
\end{abstract}

DOI: 10.1103/PhysRevX.7.031017

Subject Areas: Industrial Physics, Interdisciplinary Physics, Plasmonics

\section{INTRODUCTION}

The concept of superdirectivity has permeated the areas of physics and applied physics repeatedly since Oseen discussed the concept of "needle radiation" almost a century ago [1]. While Oseen was keenly interested in how a tiny atom might absorb a large electromagnetic wave as an equivalent photon (and, consequently, the alternate translation of his paper's title as "pinprick" radiation might make more historical sense), the reciprocal problem of transmitting a needle-like radiation pattern from a small source has stimulated many physics and, possibly more, engineering discussions. The role of superdirectivity in radio astronomy and in particle physics was discussed by Casimir [2] and Wheeler [3]. They, too, emphasized the possibility that the effective receiving cross section of a radio telescope or an atom could be extremely large in comparison with its physical size. This concept has been demonstrated more recently with plasmonic particles whose strong reactive scattering components extend to large distances and redirect the power passing through a large area of an incoming plane wave and force it to flow towards the scatterer [4-7].

Engineering the emission of electromagnetic fields from finite sources was intensely studied in the 1940s and 1950s

*Richard.Ziolkowski@uts.edu.au, ziolkowski@ece.arizona.edu

Published by the American Physical Society under the terms of the Creative Commons Attribution 4.0 International license. Further distribution of this work must maintain attribution to the author(s) and the published article's title, journal citation, and DOI. soon after Oseen's publication. Both end-fire (maximum radiated power is oriented along the array direction) [8] and broadside (maximum radiated power is oriented perpendicular to the plane of the array) [9] pattern enhancements from different array configurations were considered initially. La Paz and Miller [10] purported to show that the maximum directivity from an aperture of a given size was fixed, but Bouwkamp and De Bruijn [11] correctly demonstrated that there was no theoretical limit on the directivity from an aperture of any size. Dolph realized that one could control the sidelobe levels of the pattern by properly weighting (Chebyshev polynomial tapering) the amplitudes of the element excitations [12]. Riblet [13,14] illustrated that such amplitude tapering has an associated cost of widening the mainlobe of the pattern. However, it was quickly shown by Yaru [15] that the current distribution solutions that produce superdirective beams from arrays are generally ill-posed [16]; i.e., small variations of the large positive and negative variations of the excitation amplitudes required to achieve the effect lead to its disappearance in practice. In fact, Casimir [2] and Wheeler [3] noted this practical difficulty and believed that one would never go beyond combining a dipole and a quadrupole mode together in practice. Nonetheless, this goal has been achieved with subwavelength dielectric and plasmonic particles [17-22]. There have been and continue to be many examples of optimizing the directivity from an antenna system with constraints on its various other performance characteristics to circumvent the ill-posedness of the "super" outcome [23-27].

A useful operational definition of superdirectivity, e.g., as emphasized by Hansen [28,29], is to achieve a directivity 
greater than that obtained with the same antenna configuration being uniformly excited (constant amplitude and phase). Let the radiating system be either an aperture antenna (continuous current distribution) whose effective area is $A_{\text {eff }}$ or an array of radiating elements (set of discrete currents) distributed in $A_{\text {eff }}$. If the total efficiency (i.e., taking into account the material losses, mismatch losses, polarization mismatch,...) of the system is $e_{\text {total }}$, then its maximum gain $G_{\max }$ is related to its maximum directivity $D_{\max }$ as $G_{\max }=e_{\text {total }} \times D_{\max }$. Thus, if there are no losses, then the maximum directivity of the antenna system uniformly driven at the excitation wavelength $\lambda$ is fundamentally related to its effective area $A_{\text {eff }}$ as [30]

$$
D_{\max }=\frac{4 \pi}{\lambda^{2}} A_{\text {eff }} .
$$

Consequently, a larger effective aperture will provide a higher directivity.

As has been shown by a number of authors, e.g., Refs. [31-33], the fields in a region of free space outside of a spherical surface that encloses all the currents can be expanded in a series of electric and magnetic multipole fields represented by (vector) spherical harmonics (see Ref. [34] for more details). This approach has proved to be very successful for the analysis of the far-field behavior of an antenna system [30]. By taking into account both the transverse electric and magnetic modes, Harrington demonstrated that the maximum directivity from a source region as a function of the number of multipole modes, $N$, is $[35,36]$

$$
D_{\max }=N^{2}+2 N .
$$

Therefore, by exciting higher order modes, one can achieve very high directivities from a fixed source region.

These antenna results are immediately connected to the upper bounds on the total cross section associated with scattering from particles [37]. The concept of subwavelength superscattering $[38,39]$ arises from maximizing the contributions from a sufficiently large number of channels; i.e., by aligning the frequencies of higher-order resonant multipole modes, arbitrarily large total cross sections can be achieved with subwavelength structures. In fact, the ability to create highly subwavelength (electrically small) radiators and scatterers has been one of the success stories associated with metamaterials [40-42]. Moreover, recent passive and active nanoparticle studies associated with the optical theorem [43], Kerker conditions [21], and Huygens source effects [44] illustrate that combining sets of electric and magnetic multipoles leads to enhanced directivities. These effects have been demonstrated with numerous configurations [17-20,22,45]. The recent subwavelength superdirective results have shown that Harrington's original estimate that the maximum number of usable higher-order modes $N$ in a sphere of radius $r_{0}$ would be limited to $N=k r_{0} \equiv 2 \pi r_{0} / \lambda$ is no longer justifiable in general.

The concept of a transmitting antenna realizing a farfield needle radiation pattern is also intimately connected to subwavelength imaging, i.e., being able to resolve two small objects separated by subwavelength distances $[46,47]$. Moreover, superdirectivity has been shown to lead to enhanced channel capacity in multiple-input-multipleoutput (MIMO) systems [48-51]. Thus, superdirectivity concepts yet again become important as nanotechnology applications flourish and the Internet of things (IoT) comes to fruition. We would like to have electrically small, highly directive, receiving or transmitting antennas (whether they are macro, micro, or even nano) for numerous wireless applications.

There has been a significant increase in higher-directivity scattering approaches reported, but little on corresponding radiating systems. This article is timely in that it addresses two fundamental questions: Despite nearly a century of investigation, what would it really take to achieve actual needlelike radiation? Can one design an array that would eliminate sidelobes, has a high front-to-back ratio, has superdirective properties, and is not plagued by ill-posedness?

\section{FAR-FIELD RADIATED FROM A SET OF ELECTRIC AND MAGNETIC SOURCES}

As explained in Ref. [34], the electric field radiated into the far field of a combination of electric $\vec{J}_{\omega}$ and magnetic currents $\vec{K}_{\omega}$ excited at the frequency $f=\omega / 2 \pi$ can be written as

$\vec{E}_{J, \omega}^{f f}=i \omega \mu \frac{e^{i k r}}{4 \pi r} \iiint_{V} e^{-i k \hat{r} \cdot \vec{r}^{\prime}}\left\{\vec{J}_{\omega}\left(\vec{r}^{\prime}\right)-\hat{r}\left[\hat{r} \cdot \vec{J}_{\omega}\left(\vec{r}^{\prime}\right)\right]\right\} d^{3} \vec{r}^{\prime}$

$$
\vec{E}_{K, \omega}^{f f}=i \omega \varepsilon \frac{e^{i k r}}{4 \pi r} \iiint_{V} e^{-i k \hat{r} \cdot \vec{r}^{\prime}}\left\{\hat{r} \times\left[\vec{K}_{\omega}\left(\vec{r}^{\prime}\right)\right]\right\} d^{3} \vec{r}^{\prime} .
$$

The primed (unprimed) coordinates are the observation (source) point coordinates, and the wave number is $k=\omega / v$, with $v=1 / \sqrt{\varepsilon \mu}$ being the wave speed in the medium. The far-field magnetic field is simply $\vec{H}_{\omega}^{f f}=\hat{r} \times \vec{E}_{\omega}^{f f} / \eta$, where the free-space impedance $\eta=\sqrt{\mu / \varepsilon}$. These expressions represent the known facts that the far fields are transverse electromagnetic (TEM) and that they are related to the Fourier transform of the current components orthogonal to the observation direction.

Now consider the currents to be confined to the surface of a small sphere of radius $a$. Being as general as possible, the current densities then take the form

$$
\begin{aligned}
\vec{J}_{\omega}(\vec{r}) & =\left[J_{\theta}(\theta, \phi, \omega) \hat{\theta}+J_{\phi}(\theta, \phi, \omega) \hat{\phi}\right] \delta(r-a), \\
\vec{K}_{\omega}(\vec{r}) & =\left[K_{\theta}(\theta, \phi, \omega) \hat{\theta}+K_{\phi}(\theta, \phi, \omega) \hat{\phi}\right] \delta(r-a) .
\end{aligned}
$$


With the standard unit vector cross products $\hat{\theta} \times \hat{\phi}=\hat{r}$, $\hat{r} \times \hat{\theta}=\hat{\phi}, \hat{\phi} \times \hat{r}=\hat{\theta}$ - the far-field expressions become

$$
\begin{aligned}
\vec{E}_{\omega}^{f f}(\vec{r})= & i \omega \mu \frac{e^{i k r}}{4 \pi r} \int_{0}^{2 \pi} \int_{0}^{\pi} a^{2} \sin \theta^{\prime} d \theta^{\prime} d \phi^{\prime} e^{-i k \hat{r} \cdot \vec{r}^{\prime}} \\
& \times\left\{\left[J_{\theta}\left(\theta^{\prime}, \phi^{\prime}, \omega\right)+\frac{1}{\eta} K_{\phi}\left(\theta^{\prime}, \phi^{\prime}, \omega\right)\right] \hat{\theta}\right. \\
& \left.+\left[J_{\phi}\left(\theta^{\prime}, \phi^{\prime}, \omega\right)-\frac{1}{\eta} K_{\theta}\left(\theta^{\prime}, \phi^{\prime}, \omega\right)\right] \hat{\phi}\right\}, \\
\vec{H}_{\omega}^{f f}(\vec{r})= & \frac{1}{\eta} \hat{r} \times \vec{E}_{\omega}^{f f}(\vec{r}) .
\end{aligned}
$$

As explained in Ref. [34], it is clear that to achieve a Huygens source behavior, one can simply consider the contributions from either the orthogonal pair $J_{\theta}$ and $K_{\phi}$ or from its dual, $J_{\phi}$ and $K_{\theta}$. Electing the former, one has

$$
\begin{aligned}
\vec{E}_{\omega}^{f f}(\vec{r})= & i \omega \mu \frac{e^{i k r}}{4 \pi r} \int_{0}^{2 \pi} \int_{0}^{\pi} a^{2} \sin \theta^{\prime} d \theta^{\prime} d \phi^{\prime} e^{-i k \hat{r} \cdot \vec{r}^{\prime}} \\
& \times\left\{\left[J_{\theta}\left(\theta^{\prime}, \phi^{\prime}, \omega\right)+\frac{1}{\eta} K_{\phi}\left(\theta^{\prime}, \phi^{\prime}, \omega\right)\right] \hat{\theta}\right\}, \\
\vec{H}_{\omega}^{f f}(\vec{r})= & \frac{1}{\eta} \hat{r} \times \vec{E}_{\omega}^{f f}(\vec{r}) .
\end{aligned}
$$

By properly selecting those currents, we will show that the desired needle radiation can be achieved.

\section{NEEDLE RADIATION FROM CURRENTS ON A SMALL SPHERE}

First, consider the electric far-field component produced by only the $J$ source:

$$
\begin{aligned}
E_{\theta}^{J, f f}(\vec{r}, \omega)= & i \omega \mu \frac{e^{i k r}}{4 \pi r} \int_{0}^{2 \pi} \int_{0}^{\pi} a^{2} \sin \theta^{\prime} d \theta^{\prime} d \phi^{\prime} \\
& \times e^{-i k \hat{r} \cdot \vec{r}^{\prime}} J_{\theta}\left(\theta^{\prime}, \phi^{\prime}, \omega\right),
\end{aligned}
$$

which can be written immediately as

$$
E_{\theta}^{J, f f}(r, \theta, \phi, \omega)=i \omega \mu \frac{e^{i k r}}{4 \pi r} J_{0} a^{2} \mathcal{F} \mathcal{J}_{\theta}(\theta, \phi, \omega),
$$

where the Fourier transform of the normalized current density component, $\mathcal{F} \mathcal{J}_{\theta}(\theta, \phi, \omega)$, has been introduced. It defines the angular distribution or pattern of the far field, and in this article, it will be denoted as the pattern function. Setting $J_{\theta}(\theta, \phi, \omega)=J_{0} \Pi_{\theta}(\theta, \phi, \omega), \mathcal{F} \mathcal{J}_{\theta}$ is given by an integral over the unit sphere $S^{2}$, whose differential surface area $d \Omega=\sin \theta d \theta d \phi$, as

$$
\mathcal{F} \mathcal{J}_{\theta}(\theta, \phi, \omega)=\oiint_{S^{2}} d \Omega^{\prime} e^{-i k a \hat{r}(\theta, \phi) \cdot \hat{r}\left(\theta^{\prime}, \phi^{\prime}\right)} \Pi_{\theta}\left(\theta^{\prime}, \phi^{\prime}, \omega\right) .
$$

The fact that the source points are on the sphere of radius $a$ has allowed us to write $\vec{r}^{\prime}=a \hat{r}\left(\theta^{\prime}, \phi^{\prime}\right)$, while emphasizing that the observation direction $\hat{r}$ is given by the coordinates $(\theta, \phi)$. The far-field pattern function is now more clearly related to the $2 \mathrm{D}$ Fourier transform of the current density pattern $\Pi_{\theta}$ over the unit sphere.

The far-field expression (10) indicates that there are no dc components of the source excitation radiated into the far field and that the radial dependence is that of a spherical wave. The Fourier transform integral determines if there are any preferred directions into which the fields are radiated. Since we desire needle radiation along the $z$ axis, it would follow that the pattern function yields

$$
\mathcal{F} \mathcal{J}_{\theta}(\theta, \phi, \omega)=\delta(\cos \theta-1) \delta(\phi) \equiv \delta(\hat{r}-\hat{z}) .
$$

From this relation, it is immediately apparent that the currents on the sphere will have to be azimuthally symmetric with respect to the $z$ axis to achieve the desired outcome.

To proceed, several spherical harmonic relations, as reviewed briefly in Ref. [34], are employed. Since the spherical harmonics are a complete basis, one can expand the angular behavior of the theta component of the electric current density pattern on the sphere as

$$
\Pi_{\theta}(\theta, \phi)=\sum_{\ell=0}^{\infty} \sum_{m=-\ell}^{\ell} c_{\ell m} Y_{\ell m}(\theta, \phi),
$$

where $Y_{\ell m}$ is the spherical harmonic of degree $\ell$ and order $m$ and the coefficients are given explicitly as

$$
c_{\ell m}=\oiint_{S^{2}} \Pi_{\theta}(\theta, \phi) Y_{\ell m}^{*}(\theta, \phi) \sin \theta d \theta d \phi,
$$

with the asterisk denoting the complex conjugate operation. Combining these expressions with the spherical harmonic expansion of the exponential term in the pattern function integral, the pattern function itself becomes

$$
\begin{aligned}
& \mathcal{F} \mathcal{J}_{\theta}(\theta, \phi, \omega) \\
& =\oiint_{S^{2}} d \Omega^{\prime}\left\{4 \pi \sum_{\ell=0}^{\infty} \sum_{m=-\ell}^{\ell}(-i)^{\ell} j_{\ell}(k a) Y_{\ell m}(\theta, \phi) Y_{\ell m}^{*}\left(\theta^{\prime}, \phi^{\prime}\right)\right\} \\
& \quad \times \sum_{\ell^{\prime}=0}^{\infty} \sum_{m^{\prime}=-\ell^{\prime}}^{\ell^{\prime}} c_{\ell^{\prime} m^{\prime}} Y_{\ell^{\prime} m^{\prime}}\left(\theta^{\prime}, \phi^{\prime}\right) .
\end{aligned}
$$

Recombining terms to take advantage of spherical harmonic identities and orthogonality properties and resetting the indices to simplify the notations, the pattern function becomes 


$$
\begin{aligned}
\mathcal{F} \mathcal{J}_{\theta}(\theta, \phi, \omega)= & 4 \pi \sum_{\ell^{\prime}=0}^{\infty} \sum_{m^{\prime}=-\ell^{\prime}}^{\ell^{\prime}} c_{\ell^{\prime} m^{\prime}} \\
& \times \sum_{\ell=0}^{\infty} \sum_{m=-\ell}^{\ell}(-i)^{\ell} j_{\ell}(k a) Y_{\ell m}(\theta, \phi) \\
& \times \oiint_{S^{2}} d \Omega^{\prime} Y_{\ell m}^{*}\left(\theta^{\prime}, \phi^{\prime}\right) Y_{\ell^{\prime} m^{\prime}}\left(\theta^{\prime}, \phi^{\prime}\right) \\
= & 4 \pi \sum_{\ell=0}^{\infty} \sum_{m=-\ell}^{\ell}(-i)^{\ell} c_{\ell m} j_{\ell}(k a) Y_{\ell m}(\theta, \phi) .
\end{aligned}
$$

Consequently, taking into account the need for azimuthal symmetry in the currents from Eq. (12) and the spherical harmonic completeness relation given in Ref. [34], one sets

$$
c_{\ell m}=\frac{1}{4 \pi} \sqrt{\frac{2 \ell+1}{4 \pi}} \frac{i^{\ell}}{j_{\ell}(k a)} \delta_{m 0}
$$

to obtain the desired explicit needle radiation result:

$$
\begin{aligned}
\mathcal{F J}_{\theta}(\theta, \phi, \omega) & =\sum_{\ell=0}^{\infty} \sum_{m=-\ell}^{\ell} \sqrt{\frac{2 \ell+1}{4 \pi}} Y_{\ell m}(\theta, \phi) \delta_{m 0} \\
& =\sum_{\ell=0}^{\infty}\left[\frac{2 \ell+1}{4 \pi}\right] P_{\ell}(\cos \theta) \delta(\phi) \\
& \equiv \delta(\hat{r}-\hat{z}),
\end{aligned}
$$

where $P_{\ell}$ is the Legendre function of degree $\ell$. It is emphasized that the process leading to Eq. (16) is not the usual invocation that $k a$ is small, with the subsequent expansion of the exponential to generate the standard multipole expansion. Rather, the desired needle radiation has been obtained directly by using the exact multimode spherical harmonic expansions and summing over all of the azimuthally symmetric modes on the sphere.

An interesting outcome of this result is the fact that the needle radiation was obtained only with electric currents (or by duality, the same outcome is obtained with only magnetic currents). One would then automatically obtain a factor of 2 in amplitude of the needle peak value in the far field if the magnetic (electric) currents were also included (see Ref. [34]) with $K_{0}=\eta J_{0}\left(J_{0}=K_{0} / \eta\right)$. On the other hand, how does the directivity behave as a function of the number of modes?

In particular, consider the pattern function for $N$ modes,

$$
\mathcal{P}_{N}(\theta)=\sum_{\ell=0}^{N}\left[\frac{2 \ell+1}{4 \pi}\right] P_{\ell}(\cos \theta) .
$$

The directivity for the Huygens current needle radiation approximation with $N$ pairs of modes, i.e., when $N$ modes of both the electric and magnetic currents are present on the sphere, is then

$$
D(\theta, \phi)=\frac{2(1+\cos \theta)^{2} \times \mathcal{P}_{N}^{2}(\theta)}{\int_{0}^{\pi}(1+\cos \theta)^{2} \times \mathcal{P}_{N}^{2}(\theta) \sin \theta d \theta} .
$$

The directivity patterns (in $\mathrm{dB}$ ) for several numbers of modes are given in Fig. 1. A comparison of the maximum directivity of the needle radiation for the Huygens current case and the electric-only current case as functions of the number of modes $N$ is presented in Fig. 2. Both of these cases are then compared to the Harrington limit (2) in Fig. 3.

One finds from Fig. 3 that when $N$ modes of the electric currents are considered, i.e., without the Huygens factor $(1+\cos \theta)^{2}$ included, the maximum directivity grows as predicted by Eq. (2), i.e., as $\sum_{\ell=1}^{N}(2 \ell+1)=N^{2}+2 N$, with the $N=0$ term not being included since it is a leftover from the potential formulation used to obtain the far-field representations (see Ref. [34]) and is actually not radiated

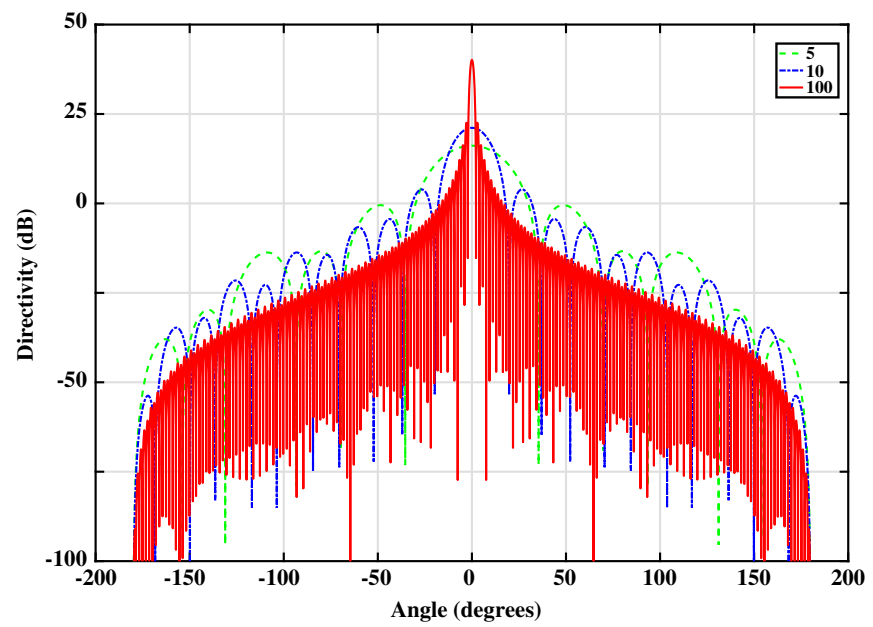

FIG. 1. Directivity pattern (dB) of the Huygens current needle radiation limited to $N$ modes.

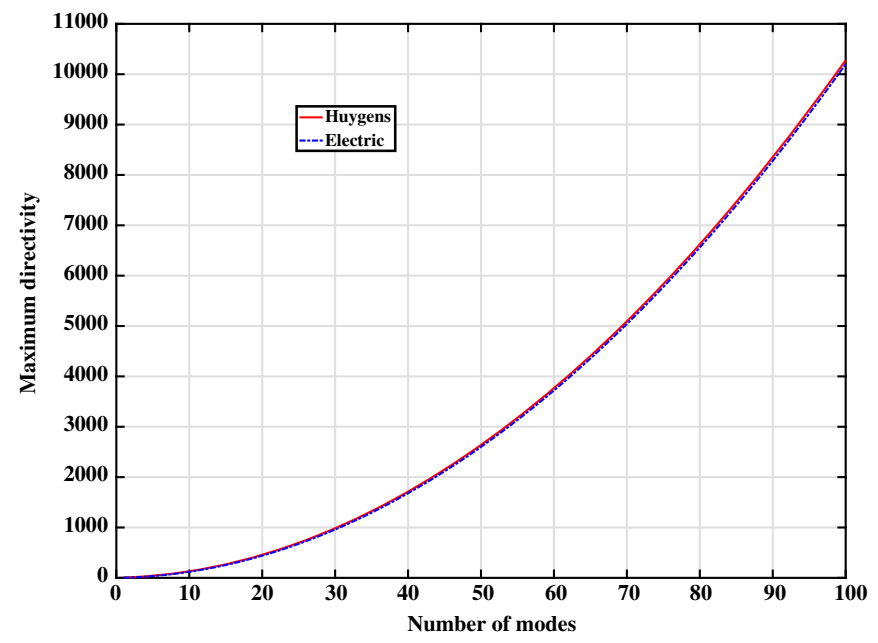

FIG. 2. Comparison of the maximum directivity of the needle radiation for the Huygens current and the electric-only current cases limited to $N$ modes. 


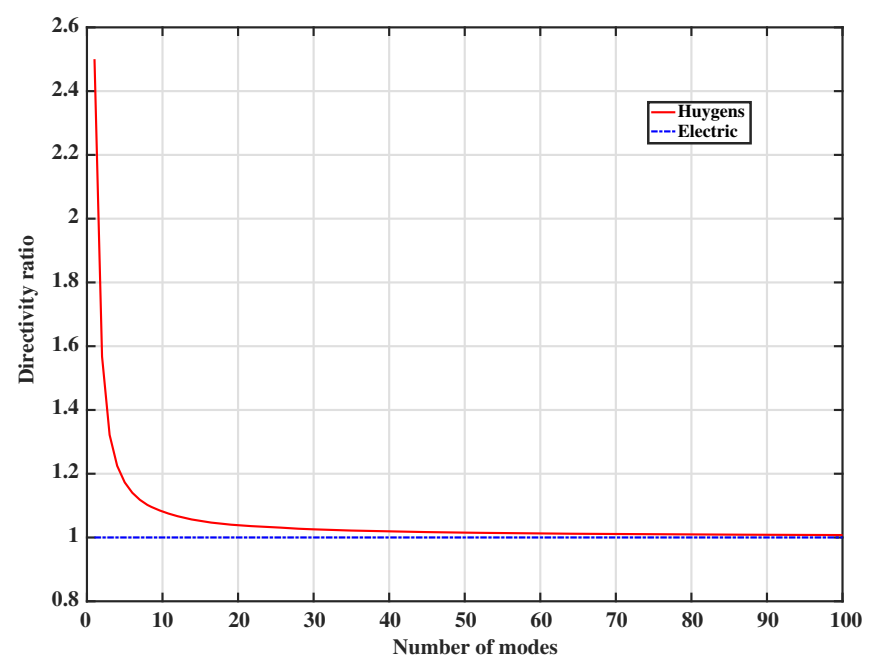

FIG. 3. Comparison of the ratio of the maximum directivity of the needle radiation for the Huygens current and the electric-only current cases, limited to $N$ modes, to the Harrington limit (2).

as an electromagnetic wave (the dipole term, $\ell=1$, is the lowest-order radiated mode). On the other hand, while the directivities are apparently the same in Fig. 2 when the Huygens factor is present, Fig. 3 also shows that there is a very noticeable difference when only the first few modes are taken into account. The Huygens case value is much larger. This outcome was confirmed by hand for the first few modes. Nevertheless, the Huygens case's maximum directivity eventually converges to Harrington's limit (2) as $N$ becomes quite large. This was a totally unexpected result. It was originally anticipated that the hole in the directivity in the back direction would give the Huygens source the advantage for all $N$. However, as shown in Fig. 1, the large increase in the number of sidelobes as $N$ increases basically fills in the back-direction hole, and the initial advantage is lost.

While the peak values of the directivity increase quadratically as $N$ increases, the corresponding full-width-athalf-maximum (FWHM) values of the main beam are given in Fig. 4. One can clearly see that the needle-like behavior is emerging as $N$ increases. The width of the main beam decreases rapidly as the peak directivity increases.

In contrast to the known 2D planar aperture or array approaches to high directivity, some of which are discussed in Ref. [34], Eq. (16) demonstrates that currents on a 3D sphere can achieve true needle radiation in theory. Nevertheless, again examining the directivity patterns in Fig. 1, the increasing numbers of sidelobes illuminate yet another issue. While the sidelobe levels are decreasing as $N$ increases and the outcome would be the eventual achievement of the true needle result, they are impressively present in large numbers for a finite number of modes. This sidelobe behavior is very undesirable for many applications, especially if only a low number of modes were excited. Moreover, when one examines the amplitudes of

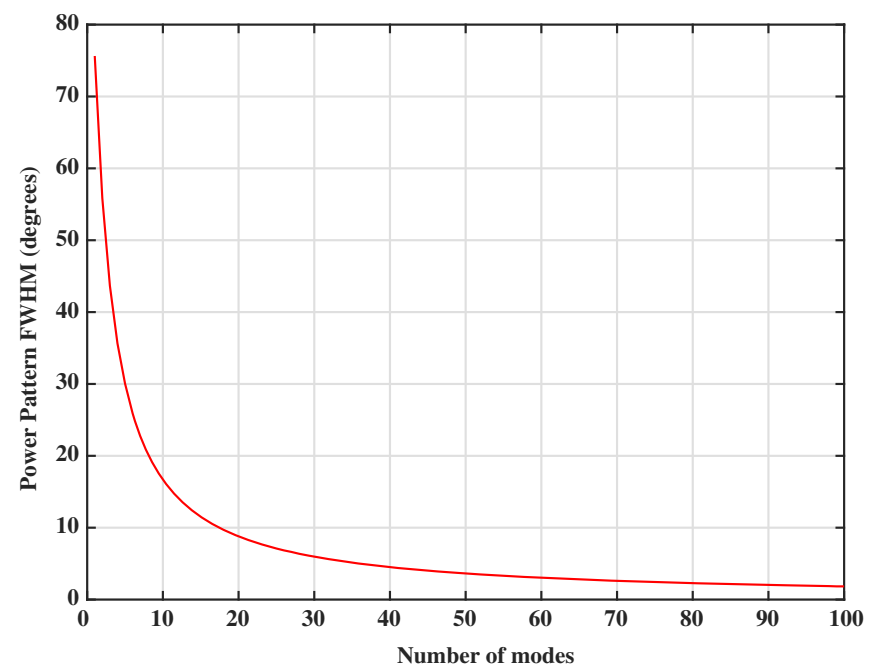

FIG. 4. FWHM values of the directivity pattern of the Huygens current needle radiation limited to $N$ modes.

the coefficients Eq. (15) as $N$ increases, one finds problems with these very-high-order modes. In fact, the same illposedness problems encountered with the planar arrays arise but in a slightly different manner.

One finds that the original considerations by Harrington about the sphere size and the number of modes $[35,36]$ actually play a significant and related role in this case. If one restricts the number of modes to $N$, i.e., to the pattern function $\mathcal{P}_{N}(\theta)$, and selects the electrical size of the sphere to be $k a=N$, then the coefficients in the current expansion are manageable. On the other hand, if one tries to realize the needle radiation result from an electrically small sphere, i.e., from a sphere with $k a \leq 1, k a=1$ being the Wheeler radiansphere whose radius is $a=\lambda / 2 \pi[52,53]$, the usually restrictive large current amplitudes occur quite quickly as the index $n$ of the coefficient $1 / j_{n}(k a)$ increases. This is clearly illustrated in Fig. 5. One observes the oscillations of

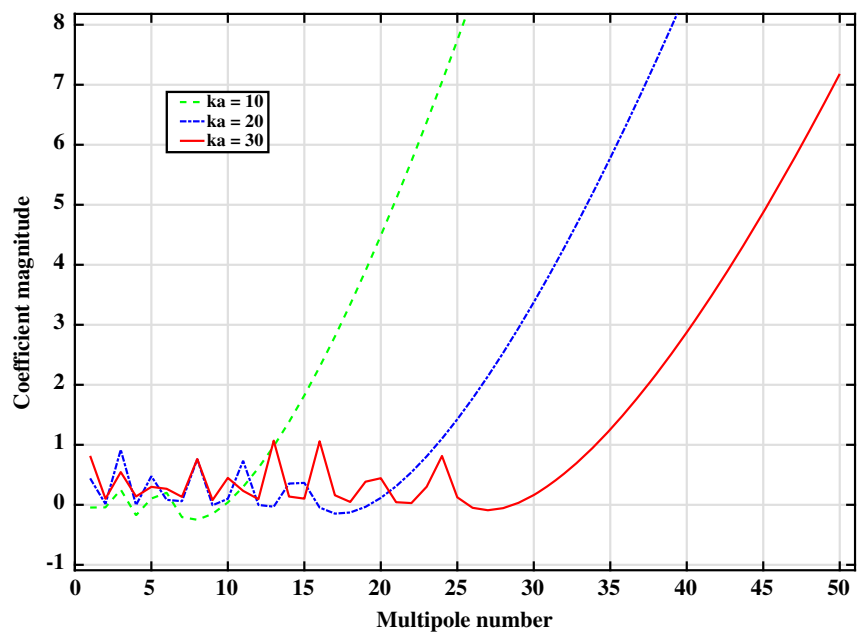

FIG. 5. Needle radiation expansion coefficients for various electrical sizes of the sphere, $k a$, as functions of the number of azimuthally symmetric modes, $N$. 
the amplitudes for small mode numbers and the exponential growth of the coefficients once the mode number exceeds the electrical size of the sphere. Thus, in practice, one could only hope to approach a "true" needle effect from an increasingly larger number of tailored currents on an increasingly larger sphere, basically in agreement with Eq. (1).

\section{NEEDLE-LIKE RADIATION FROM AN ARRAY OF DIPOLE-CONSTRUCTED HUYGENS MULTIPOLES}

Obtaining sets of specified current distributions on an electrically small 3D object with curvature to realize an approximate needle radiation even for a few modes is also, unfortunately, a nontrivial task. Moreover, one would nevertheless desire a planar or conformal array or at least a thin stack of planar-radiating elements on a mobile platform for any practical application. Thus, a discrete radiating aperture associated with a simple set of radiators to achieve high directivity, like what is obtained with now commonly used phased arrays, remains desirable. Can something useful be achieved in practice?

As the historical discussion indicated, it is well known that there are generally fundamental trade-offs in the patterns generated by a discrete array between the main beam width and the sidelobe levels [30]. One knows that a uniformly driven aperture generally produces the maximum directivity, amplitude tapering of the array elements provides control of the sidelobe levels, and phasing between the elements yields the capability to steer the direction of the main beam. Examples of the directivity obtained from distributions of electric and magnetic currents on a planar disk and from a circular array of Huygens dipole sources are given in Ref. [34].

One finds that in contrast to the currents on a small sphere, those 2D current distributions do not yield the desired needle-like radiation pattern unless the disk radius becomes extremely large or hard-to-realize current distributions are employed. What can one then do to achieve needle-like radiation from a potentially realizable current distribution? Here, we explore how superdirectivity can be obtained with an end-fire array of broadside radiating Huygens multipoles.

\section{A. Dipole-based Huygens sources}

To understand more completely the Huygens source concept, a combination of elemental electric and magnetic dipole sources is considered first. With an emphasis on the $z$ axis as the preferred direction, the elemental electric and magnetic current densities of amplitude, respectively, $I_{e}$ and $I_{m}$, imposed on a pair of orthogonally oriented electric and magnetic Hertzian dipole antennas of length $\ell$ will be taken as
$\vec{J}(x, y, z)=I_{e} \ell \delta(x) \delta(y)[\mathcal{H}(z-\ell / 2)-\mathcal{H}(z+\ell / 2)] \hat{x}$,

$\vec{K}(x, y, z)=I_{m} \ell \delta(x) \delta(y)[\mathcal{H}(z-\ell / 2)-\mathcal{H}(z+\ell / 2)] \hat{y}$,

where the Heaviside function $\mathcal{H}(u)=1$ if $u>0$, and $=0$ if $u<0$. Recall that dipole antennas radiate in their broadside directions. With these current direction choices, radiated fields along the $z$ axis are thus possible. As shown in Ref. [34], if the current amplitudes are weighted properly so that $I=I_{e}=I_{m} / \eta$, one then has

$\begin{aligned} \vec{E}_{\omega}^{f f}(\vec{r}) & =i \omega \mu I \ell \frac{e^{i k r}}{4 \pi r}(1+\cos \theta)[\cos \phi \hat{\theta}-\sin \phi \hat{\phi}], \\ \vec{H}_{\omega}^{f f}(\vec{r}) & =i \omega \mu \frac{I \ell}{\eta} \frac{e^{i k r}}{4 \pi r}(1+\cos \theta)[\sin \phi \hat{\theta}+\cos \phi \hat{\phi}] .\end{aligned}$

It is immediately apparent that the cardioid pattern associated with a Huygens source is attained and that the field is null along the negative $z$ axis as expected, where $\theta=\pi$, $\phi=0$. The directivity is then straightforwardly calculated to be

$$
D(\theta, \phi)=\frac{4 \pi r^{2} \vec{S}(\vec{r}) \cdot \hat{r}}{P_{\mathrm{tot}}^{\mathrm{rad}}}=\frac{3}{4}(1+\cos \theta)^{2} .
$$

Therefore, the maximum directivity of the electric-magnetic dipole pair $(N=1)$, which is along the positive $z$ axis, where $\theta=0$, is 3 , twice the value of either dipole alone, confirming the Harrington result (2): $D_{\max }=1^{2}+2 \times 1=3$.

Huygens source antennas have been achieved in practice in both electrically small [54-57] and larger [58,59] packages. They have been recognized as an important research direction for IoT applications [60]. Huygens metasurfaces have already played a significant role in antenna and scattering configurations [61-63]. How, then, does one achieve a Huygens behavior with yet higher directivity?

\section{B. Multipole-based Huygens sources}

In an extension of Uzkov's results [64], it has been demonstrated in a series of articles on end-fire arrays, e.g., Refs. [65-72], that an array of electric elements achieves its maximum directivity in its end-fire direction when the separation distance between the element pairs goes to zero. These dense packing and end-fire concepts have been demonstrated experimentally for moderately small separation distances as well. Moreover, it has been shown that a dense end-fire array of dipole Huygens sources, i.e., electric and magnetic dipole pairs in an end-fire configuration, will produce the highest possible directivity associated with dipole radiating elements [70]. As an extension 


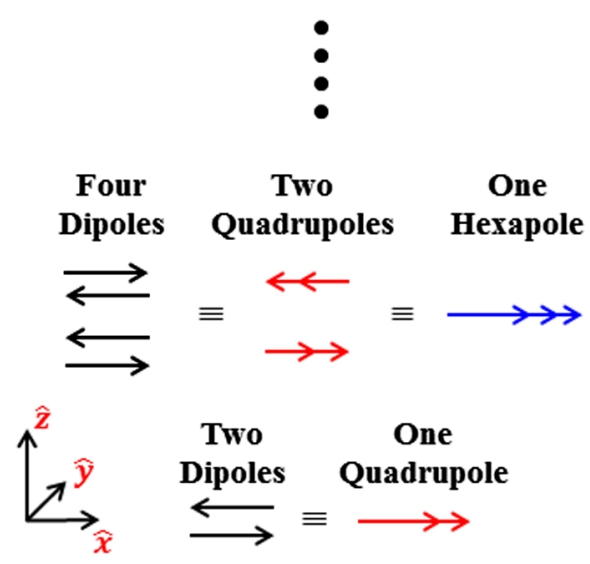

\section{Assemblage of the broadside radiating} electric and magnetic multipoles

FIG. 6. Assemblage of the broadside radiating multipoles.

of those results, let us consider a compact end-fire array of broadside radiating Huygens multipole sources.

As noted by Harrington [73], one can use alternating pairs of dipole current elements, as one does with alternating sets of charges to achieve electrostatic or magnetostatic multipoles, to produce higher-order electromagnetic multipoles. Again, consider the electric (magnetic) multipoles to be oriented along the $x$ axis ( $y$ axis). As discussed in Ref. [34] and as depicted in Fig. 6, the electric (magnetic) multipoles are obtained by properly arranging combinations of electric (magnetic) dipoles to be compactly spaced along the $z$ axis, i.e., to have an electrically small distance $\Delta$ between each of them, and to have the appropriate orientations with respect to each other. The resulting electric and magnetic multipoles are then combined together, as depicted in Fig. 7, to form the Huygens multipole end-fire array. Accounting for all of the constituent electric (magnetic)

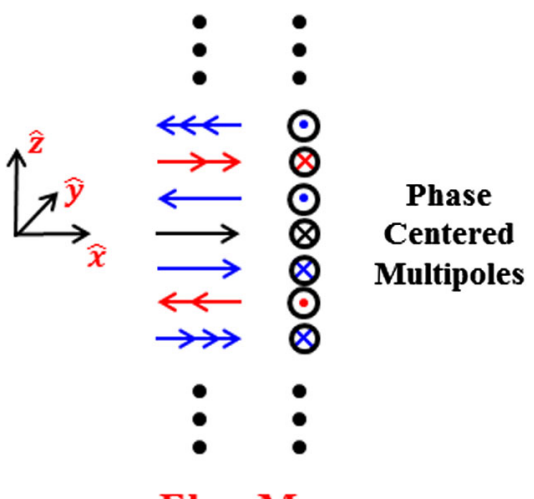

\section{Elec Mag}

\section{Huygens Multipole \\ End-fire Array}

FIG. 7. End-fire array of the assembled broadside radiating multipoles. dipoles, it has a length $2 \times N \Delta$ but with all of the resulting multipoles being centered on the zeroth element, i.e., the simple electric (magnetic) dipole. Finally, as was done with the sphere-based currents, adding the resulting electric (magnetic) multipole fields together with the simple coefficient weightings, $1 /\left\{n ![(-2 i) k \Delta]^{n}\right\}$, one obtains the pattern function

$$
\begin{aligned}
\mathcal{F J}_{z x}(\theta, \phi, \omega) & \approx \sum_{n=0}^{N}(\cos \theta)^{n}=\left\{\frac{1-\cos ^{N+1} \theta}{1-\cos \theta}\right\} \\
& =\mathcal{P}_{N}(\theta)
\end{aligned}
$$

for $N$ multipole elements. Using L'Hopital's rule, the maximum of Eq. (24) occurs along the $z$ axis at $\theta=0$, i.e., $\mathcal{P}_{N}^{\max } \sim(N+1)$. Then, arranging the electric and magnetic current moments so that they are balanced with $I_{m}=\eta I_{e}=I$, one obtains, for the far fields of $N$ electric and magnetic multipole pairs,

$$
\begin{aligned}
\vec{E}_{\omega}^{f f}(\vec{r}) & =i \omega \mu I \ell \frac{e^{i k r}}{4 \pi r}(1+\cos \theta) \mathcal{P}_{N}(\theta)[\cos \phi \hat{\theta}-\sin \phi \hat{\phi}] \\
\vec{H}_{\omega}^{f f}(\vec{r}) & =i \omega \mu \frac{I \ell}{\eta} \frac{e^{i k r}}{4 \pi r}(1+\cos \theta) \mathcal{P}_{N}(\theta)[\sin \phi \hat{\theta}+\cos \phi \hat{\phi}]
\end{aligned}
$$

Thus, the directivity again takes the form

$$
D(\theta, \phi)=\frac{2(1+\cos \theta)^{2} \times \mathcal{P}_{N}^{2}(\theta)}{\int_{0}^{\pi}(1+\cos \theta)^{2} \times \mathcal{P}_{N}^{2}(\theta) \sin \theta d \theta},
$$

where the factor of 2 appears from the equal contributions of the balanced electric and magnetic Huygens multipoles.

The directivity patterns (in $\mathrm{dB}$ ) for several number of Huygens multipoles arranged compactly along the $z$ axis are given in Fig. 8. A polar plot of the $N=1000$ case is shown in Fig. 9. From both figures, one clearly sees that the Huygens source behavior has been obtained and, as the number of higher-order modes is increased, that the directivity approaches a needle-like Huygens behavior in which the sidelobes have been completely eliminated. Thus, the desired needle-like radiation from an array has been demonstrated.

This behavior is further confirmed in Figs. 10 and 11. While they illustrate the needle-like behavior, they also indicate that this desirable performance, in contrast to the sphere result, is slow to evolve. Referring to Fig. 10, it is confirmed that while the peaks of the power patterns increase as $2(N+1)$, the maximum directivity only increases linearly as $N$ becomes large, approximately as $1.5 \mathrm{~N}$. Moreover, the decrease of the angular FWHM of the power pattern begins to slow noticeably as $N$ becomes quite large. This is emphasized further in Fig. 12, which presents the directivity patterns of the needle and the multipole 


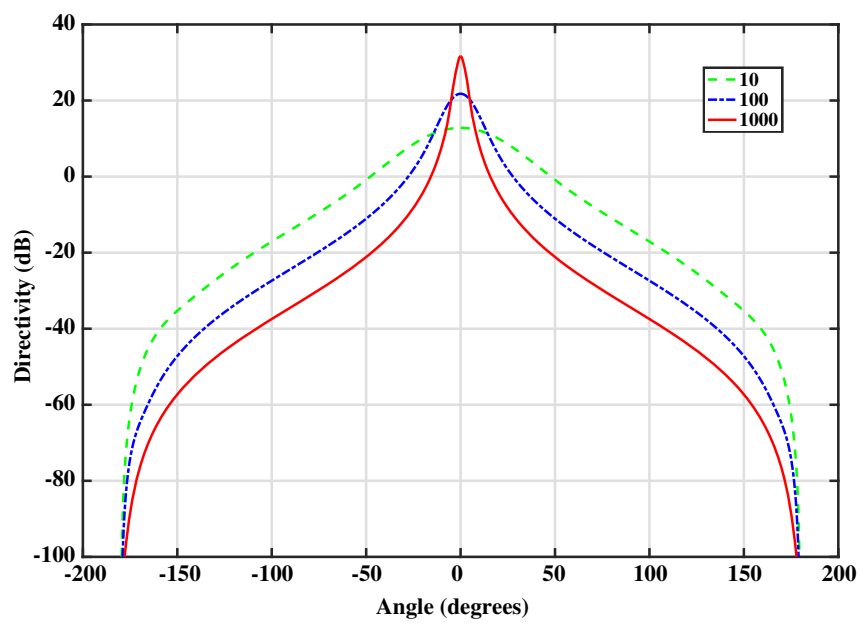

FIG. 8. Directivity pattern $(\mathrm{dB})$ attained with $N$ Huygens multipoles arranged compactly along the $z$ axis.

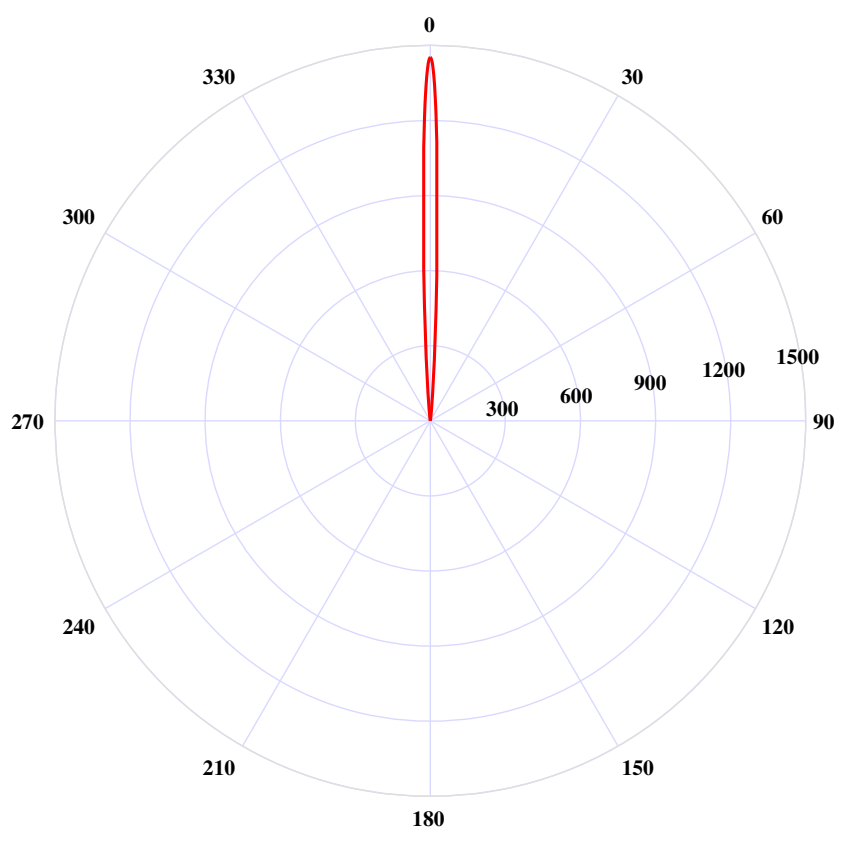

FIG. 9. Polar plot of the directivity produced by 1000 Huygens multipoles arranged compactly along the $z$ axis.

antennas for a finite number of terms adjusted for the larger number of multipoles needed to recover the maximum obtained with a much smaller number of needle terms. While it does take more terms, the multipole antenna does recover the needle behavior without the sidelobe issues.

Furthermore, considering the coefficient weightings, one does not encounter the exponential blowup associated with the sphere results. This outcome is clearly illustrated in Fig. 13, where the coefficient amplitudes for different electrical spacings (i.e., $k \Delta$, where $\Delta$ is the physical distance between the elements) between the multipoles are compared for variable numbers of multipoles. The results are different from the conventional arguments

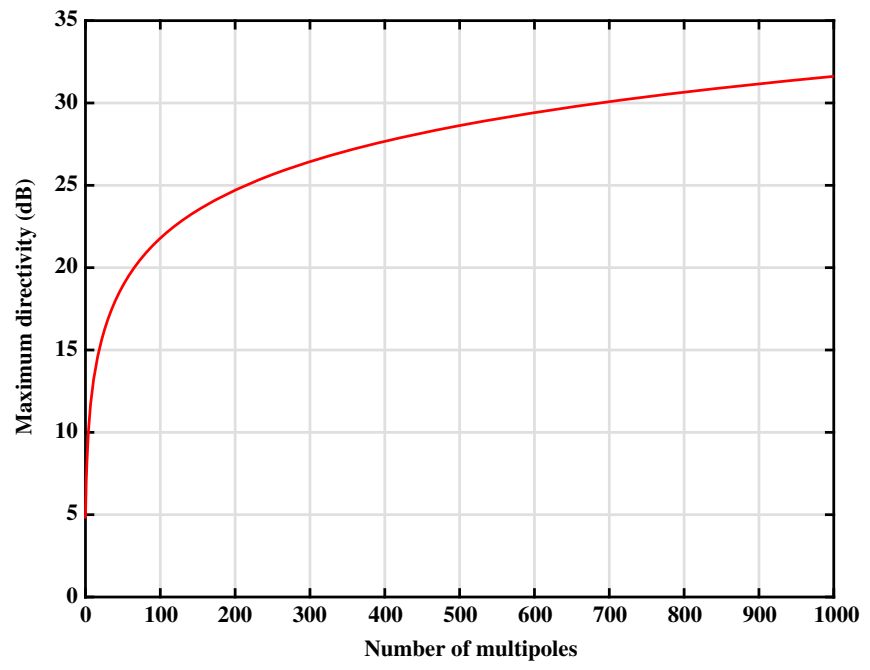

FIG. 10. Maximum directivity (dB) attained with $N$ Huygens multipoles arranged compactly along the $z$ axis.

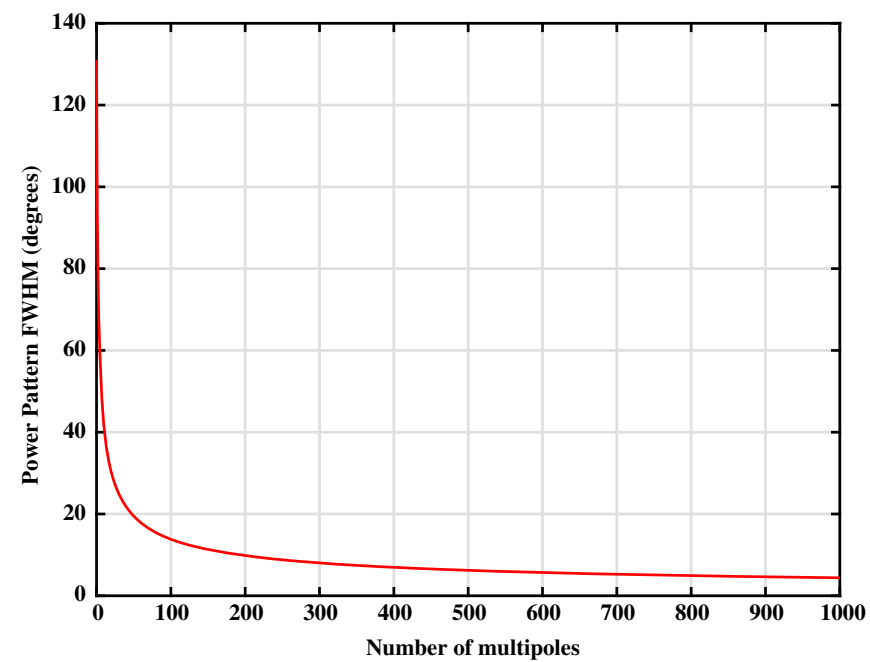

FIG. 11. FWHM values of the power patterns generated by $N$ Huygens multipoles arranged compactly along the $z$ axis.

because the $n$th multipole amplitude is the inverse of the product of the factorial terms $n$ ! and $(k \Delta)^{n}$. This means the coefficient magnitudes first increase algebraically with $n$, but they then reach a tractable maximum (as long as the multipole index is not exceedingly large, which it would not be in a realistic antenna) and start to decrease as the factorial term becomes dominant. Thus, one could hope to generate needle-like behavior in practice. These results suggest that advancing to a few Huygens multipole pairs from the simple Huygens dipole pair can significantly improve the directivity associated with a compact system. In fact, it proves that one is not bounded by the simple dipole pair, which disproves previous conclusions (see, e.g., Ref. [74]). 


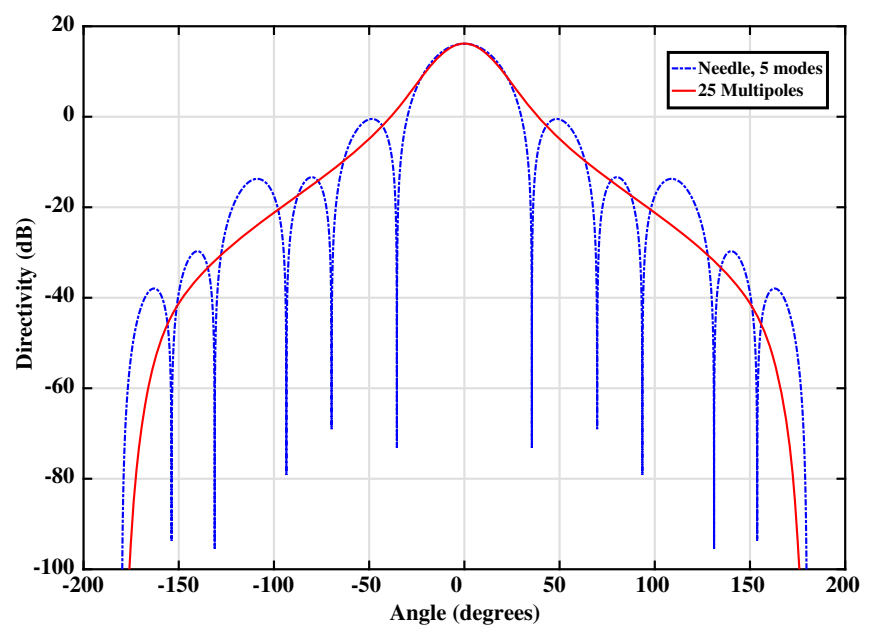

FIG. 12. Comparison of the directivity patterns $(\mathrm{dB})$ of the needle and the multipole antennas.

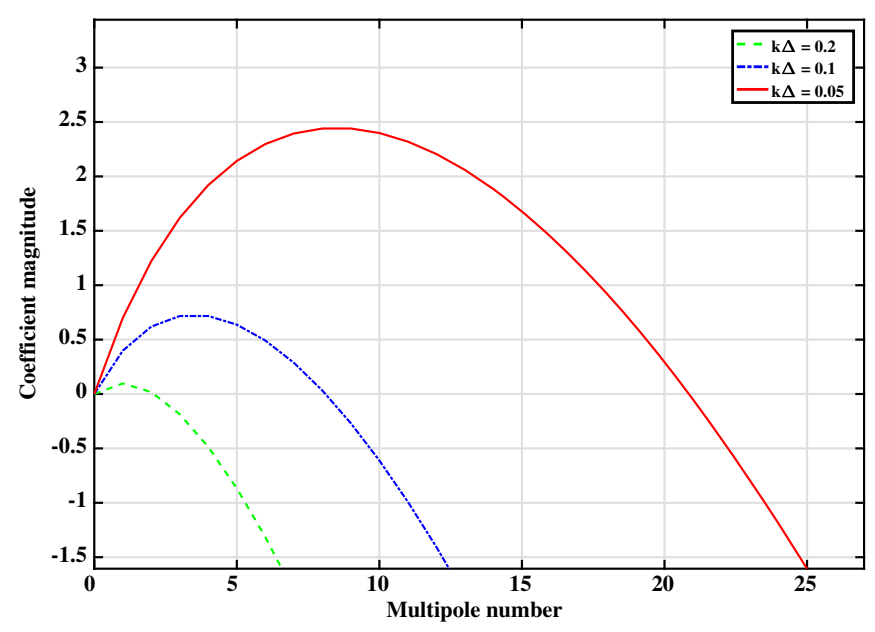

FIG. 13. Needle radiation expansion coefficients for various electrical sizes of the sphere, $k a$, as functions of the number of azimuthally symmetric modes, $N$.

Even achieving directivities that are an order of magnitude larger than an electrically small dipole from a similar footprint holds many potential benefits for future IoT wireless and mobile platform-based communication and sensor devices. Moreover, the amplitudes needed to realize the outcome are well-posed and reasonable. In practice, the desired needle-like pattern outcome could be realized by developing a compact antenna constructed as a stack of thin layers, each layer having the proper elements (dipoles or other structures) to realize the requisite higher-order multipole. The thickness of the layers would be subwavelength to ensure that $k \Delta$ is small.

As shown in Fig. 14, a parasitic stack of high-permittivity annular dielectric resonator elements could be tuned to produce the desired electric and magnetic multipoles appropriate for each layer at the same frequency and with the

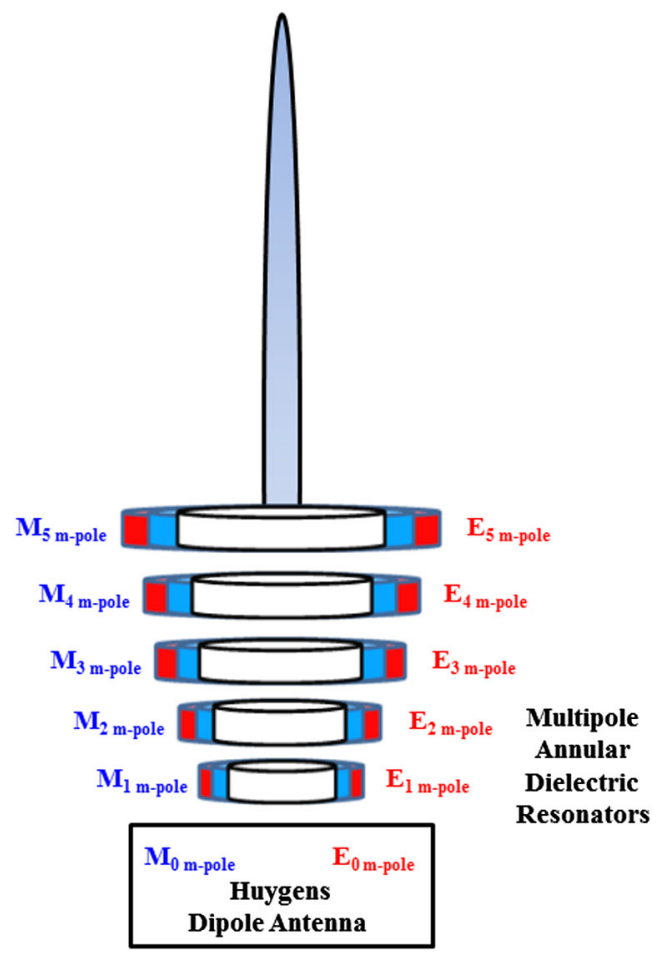

FIG. 14. Dielectric resonator stack approach to realize the Huygens multipole array.

desired broadside radiating fields. These elements would then be assembled into the end-fire array configuration as depicted. This dielectric resonator antenna (DRA)-based system is currently being explored for experimental validation of the Huygens multipole end-fire array concept.

Another approach, particularly suited for nano-antennas, would be to have a multilayered, subwavelength-size, resonant core-shell particle configuration in which the electric and magnetic multipole modes were simultaneously excited and their resonance frequencies adjusted by the geometry and material values to be coincident. While this was accomplished at the dipole level (e.g., Ref. [44]), discussions about the relationship between the quality factor and directivity [27] remind us that the higher-order multipoles will have narrower bandwidths and, hence, may be quite sensitive to their design parameters. Nonetheless, a two-dimensional version of this multilayer concept, as depicted in Fig. 15, has been verified [75]. Yet another technique would be to employ a similar number of (single) resonant core-shell particles, each producing one of the requisite multipole fields and then aligned and excited in an end-fire configuration.

All of these arrangements are intimately related to and supported by the near-field resonant parasitic (NFRP) metamaterial-inspired engineering of electrically small antennas paradigm [76], which has successfully produced a large variety of multifunctional compact systems. For instance, with one of the Huygens dipole sources already realized (see, e.g., Ref. [56]), any one of the 


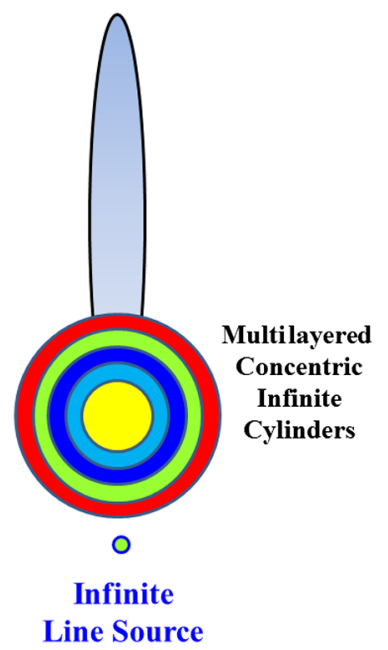

FIG. 15. Two-dimensional version of a coated nanoparticle realization of the multipole effects.

aforementioned "arrays" of NFRP electric and magnetic multipole elements could be driven at RF and microwave frequencies. This system is highlighted in Fig. 14. Moreover, as noted in the earlier discussions, the particle approaches have been demonstrated for various individual multipole orders at optical frequencies; hence, their combinations are also very realizable. Therefore, these compact Huygens multipole end-fire arrays of NFRP elements would overcome the usual argument that superdirectivity cannot be achieved because of the ill-posedness encountered with the currents typically associated with its synthesis.

\section{SIMULATED HUYGENS MULTIPOLE END-FIRE ARRAY RESULTS}

To confirm these analytical superdirectivity results and to set the stage for future experimental efforts, simulations of the Huygens multipole end-fire array were performed with the commercial method of moments code NewFasant (see Ref. [34]) [77]. It is the only commercial code (to the best of our knowledge) that has multipole sources built into it. The simulation parameters used to obtain the results were a $3.0 \mathrm{MHz}$ excitation source $\left(\lambda_{0}=100.0 \mathrm{~m}\right), 10.0 \mathrm{~cm}$ long dipoles, with $\Delta=0.1 \mathrm{~cm}$ spacings between them. The electric and magnetic dipole Huygens source was first created, and the Huygens quadrupole source was constructed with it. The result was then used to create the Huygens hexapole source and so on until the Huygens duodeca-multipole was created. Finally, these six multipoles, $n=0,1, \ldots, 5$, were combined together in the desired end-fire configuration as illustrated in Fig. 7. The overall length of this $N=5$ Huygens multipole end-fire array is $1.0 \mathrm{~cm}$ (i.e., $2 \times N \Delta=10^{-4} \lambda_{0}$ ).

The simulated 3D directivity patterns for the Huygens dipole ( $N=0$ multipole) and hexapole $(N=2$ multipole) antennas and for the composite Huygens multipole end-fire array, which combines the first six Huygens multipoles, $N=0$ to $N=5$, are shown in Figs. 16-18, respectively. The Huygens behavior in all of these cases is immediately observed. The Huygens multipole array result in Fig. 18 illustrates the absence of any sidelobes and the beginning of the needle-like behavior.

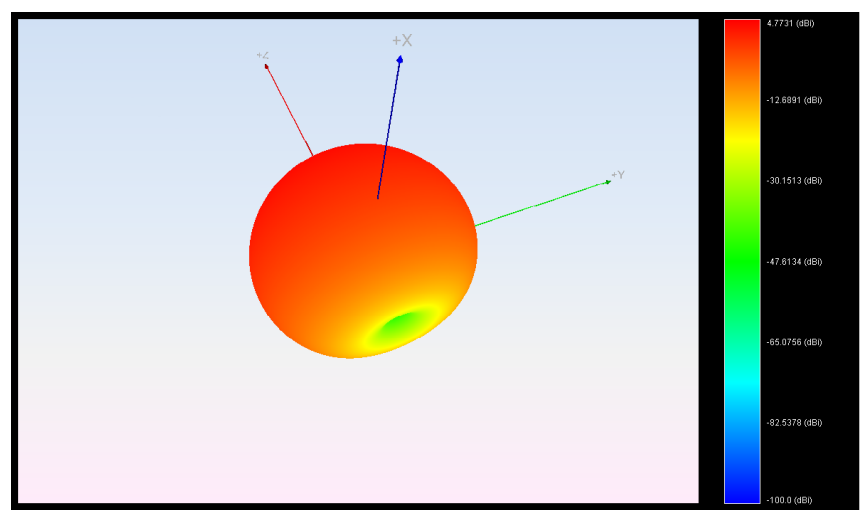

FIG. 16. NewFasant predicted 3D directivity pattern for the Huygens dipole $(N=0)$ antenna.

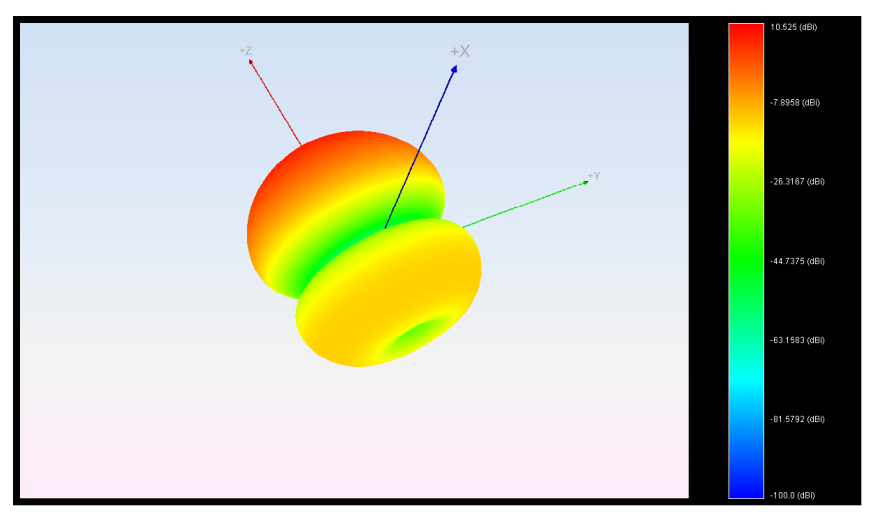

FIG. 17. NewFasant predicted 3D directivity pattern for the Huygens $N=2$ multipole antenna.

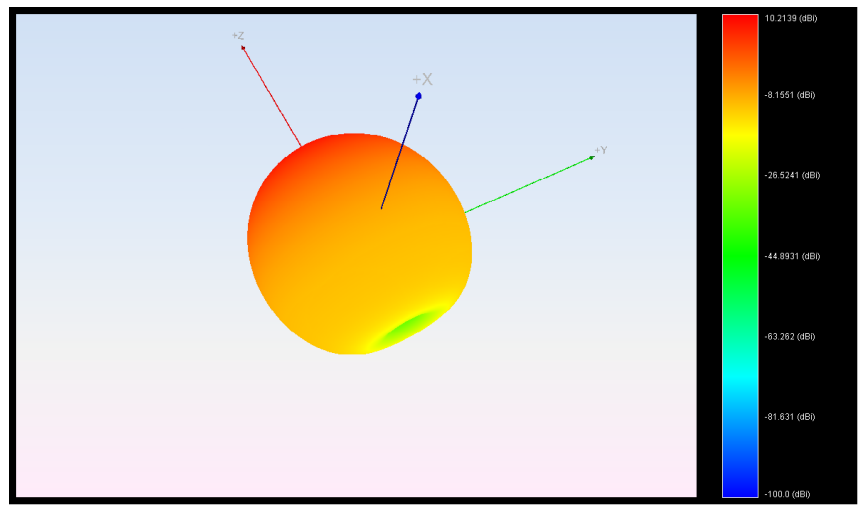

FIG. 18. NewFasant predicted 3D directivity pattern for the Huygens multipole end-fire array, which combines the first six multipoles: $N=0$ to $N=5$. 


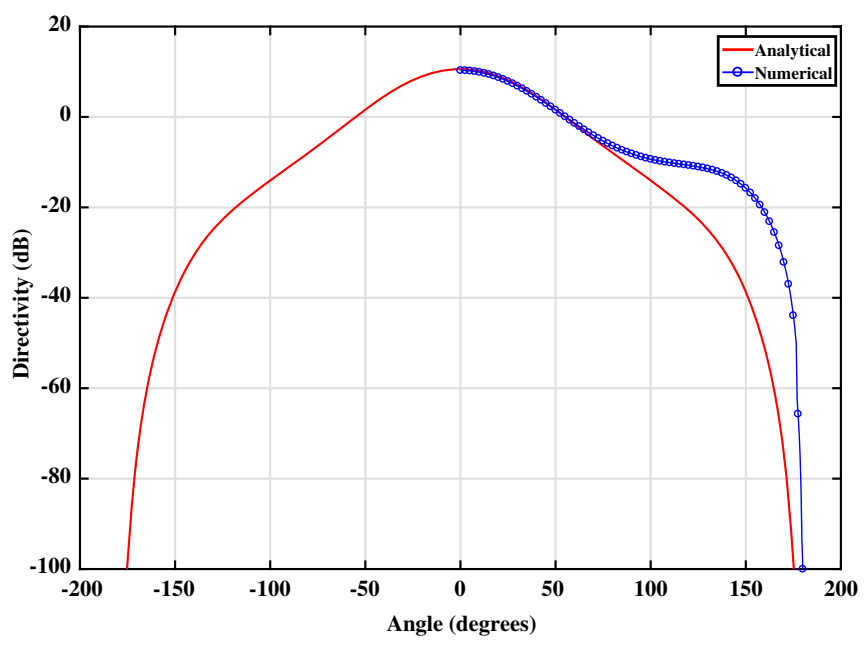

FIG. 19. Comparison of the analytically and numerically calculated directivities for the Huygens multipole end-fire array, including the dipole through the $N=5$ multipoles.

The 2D directivity pattern for the Huygens multipole end-fire array predicted numerically by NewFasant is compared with the analytical result (23) for the first six multipoles in Fig. 19. No coefficient weights were applied to the various multipole contributions in NewFasant; only the sign of each multipole was adjusted to ensure that the maximum was in the $+z$ direction. The agreement is very good in the forward hemisphere out to very large angles. However, it begins to deteriorate in the back hemisphere where the analytical results roll off more quickly. In this region, the numerical approach deals with signals smaller than $-30 \mathrm{~dB}$ from the peak values. Without any amplitude weighting to emphasize the higher-order multipole contributions, there is not a complete cancellation of these small values amongst all of the multipole fields. The numerical results go strongly to zero in the back direction, where each numerical Huygens multipole directivity pattern itself goes to zero. Moreover, no sidelobes appear. Even without the amplitude tapering of the multipole coefficients, the net Huygens multipole end-fire array numerical results show a very desirable increase of the directivity in the forward direction with no sidelobes and an extremely large front-to-back ratio.

\section{CONCLUSION}

Oseen's predicted needle radiation from a finite source region and its even-more-directive Huygens source version were demonstrated explicitly for the first time. The normal drawbacks associated with trying to realize superdirective sources were reviewed and further clarified with this solution. It was then demonstrated that a compact end-fire array of closely spaced, broadside radiating, electric and magnetic multipoles produced a needle-like Huygens source result that had high directivity and did not suffer from the exponential growth of the expansion coefficients or from the multiple sidelobes produced by a mode-limited needle source. The results disprove many previous statements that the directivity from a Huygens dipole source was the best one could accomplish from an electrically small source region. Moreover, given the many recent multipolar nano-antenna predictions and initial macroscopic-sized Huygens source realizations, an electrically small, NFRPbased Huygens multipole end-fire array should be demonstrable in the near future.

\section{ACKNOWLEDGMENTS}

The author expresses his profound gratitude to the CEO of NewFasant, Professor Felipe Catedra of the University of Alcala, Alcala de Henares, Spain, for his kindness in providing access to the NewFasant code, for writing a couple of "How to Use NewFasant for Dummies" tutorial documents, and for actually running some initial cases for my education and benefit. This work was supported in part by the Australian Research Council Grant No. DP160102219.

[1] C. W. Oseen, Einstein's pinprick radiation and Maxwell's equations, Ann. Phys. (Berlin) 374, 202 (1922).

[2] H. B. G Casimir and G. Puppi, On supergain antennae, in Old and New Problems in Elementary Particles (Academic Press, New York, 1968), pp. 73-79.

[3] J. A. Wheeler, Semiclassical analysis illuminates the connection between potential and bound states and scattering, in Studies in Mathematical Physics: Essays in Honor of Valentine Bargmann (Princeton University Press, Princeton, New Jersey, 1976), pp. 390-391.

[4] C. F. Bohren, How Can a Particle Absorb More than the Light Incident on It?, Am. J. Phys. 51, 323 (1983).

[5] H. Paul and R. Fischer, Light Absorption by a Dipole, Sov. Phys. Usp. 26, 923 (1983).

[6] S. D. Campbell and R. W. Ziolkowski, Impact of Strong Localization of the Incident Power Density on the Nanoamplifier Characteristics of Active Coated Nano-particles, Opt. Commun. 285, 3341 (2012).

[7] S. D. Campbell and R. W. Ziolkowski, Simultaneous Excitation of Electric and Magnetic Dipole Modes in a Resonant Core-Shell Particle at Infrared Frequencies to Achieve Minimal Backscattering, IEEE J. Sel. Topics Quantum Electron. 19, 4700209 (2013).

[8] W. W. Hansen and J. R. Woodyard, A New Principle in Directional Antenna Design, Proc. IRE 26, 333 (1938).

[9] S. A. Schelkunoff, A Mathematical Theory of Linear Arrays, Bell Syst. Tech. J. 22, 80 (1943).

[10] L. La Paz and G. A. Miller, Optimum Current Distributions on Vertical Antennas, Proc. IRE 31, 214 (1943).

[11] C. J. Bouwkamp and N. G. De Bruijn, The Problem of Optimum Antenna Current Distribution, Philips Res. Rep. 1, 135 (1946).

[12] C. L. Dolph, A Current Distribution for Broadside Arrays which Optimizes the Relationship between Beam Width and Side-Lobe Level, Proc. IRE 34, 335 (1946). 
[13] H. J. Riblet, Discussion on "A Current Distribution for Broadside Arrays which Optimizes the Relationship between Beam Width and Side-Lobe Level", Proc. IRE 35, 489 (1947).

[14] H. J. Riblet, Note on the Maximum Directivity of an Antenna, Proc. IRE 36, 620 (1948).

[15] N. Yaru, A Note on Super-Gain Antenna Arrays, Proc. IRE 39, 1081 (1951).

[16] G. A. Deschamps and H. S. Cabayan, Antenna Synthesis and Solution of Inverse Problems by Regularization Methods, IEEE Trans. Antennas Propag. 20, 268 (1972).

[17] I. M. Hancu, A. G. Curto, M. Castro-Lopez, M. Kuttge, and N. F. van Hulst, Multipolar Interference for Directed Light Emission, Nano Lett. 14, 166 (2014).

[18] E. Poutrina and A. Urbas, Multipole Analysis of Unidirectional Light Scattering from Plasmonic Dimers, J. Opt. 16, 114005 (2014).

[19] W. Liu, J. Zhang, B. Lei, H. Ma, W. Xie, and H. Hu, UltraDirectional Forward Scattering by Individual Core-Shell Nanoparticles, Opt. Express 22, 16178 (2014).

[20] R. R. Naraghi, S. Sukhov, and A. Dogariu, Directional Control of Scattering by All-Dielectric Core-Shell Spheres, Opt. Lett. 40, 585 (2015).

[21] R. Alaee, R. Filter, D. Lehr, F. Lederer, and C. Rockstuhl, A Generalized Kerker Condition for Highly Directive Nanoantennas, Opt. Lett. 40, 2645 (2015).

[22] E. Poutrina and A. Urbas, Multipolar Interference for Nonreciprocal Nonlinear Generation, Sci. Rep. 6, 25113 (2016).

[23] Y. T. Lo, S. W. Lee, and Q. H. Lee, Optimization of Directivity and Signal-to-Noise Ratio of an Arbitrary Antenna Array, Proc. IEEE 54, 1033 (1966).

[24] D. K. Cheng, Optimization Techniques for Antenna Arrays, Proc. IEEE 59, 1664 (1971).

[25] D. Margetis, G. Fikioris, J. M. Myers, and T. T. Wu, Highly Directive Current Distributions: General Theory, Phys. Rev. E 58, 2531 (1998).

[26] E. Shamonina, K. H. Ringhofer, and L. Solymar, Configurations Optimizing the Directivity of Planar Arrays, AEU-Int. J. Electron. Commun. 56, 115 (2002).

[27] M. Gustafsson and S. Nordebo, Optimal Antenna Currents for Q, Superdirectivity, and Radiation Patterns Using Convex Optimization, IEEE Trans. Antennas Propag. 61, 1109 (2013).

[28] R. C. Hansen, Fundamental Limitations in Antennas, Proc. IEEE 69, 170 (1981).

[29] R. C. Hansen, Some New Calculations on Antenna Superdirectivity, Proc. IEEE 69, 1365 (1981).

[30] C. A. Balanis, Antenna Theory: Analysis and Design (John Wiley \& Sons, New York, 2016).

[31] C. J. Bouwkamp and H. B. G. Casimir, On Multipole Expansions in the Theory of Electromagnetic Radiation, Physica (Amsterdam) 20, 539 (1954).

[32] J. D. Jackson, Classical Electrodynamics (Wiley, New York, 1962).

[33] A. J. Devaney and E. Wolf, Multipole Expansions and Plane Wave Representations of the Electromagnetic Field, J. Math. Phys. (N.Y.) 15, 234 (1974).

[34] See Supplemental Material at http://link.aps.org/ supplemental/10.1103/PhysRevX.7.031017 for details and examples clarifying the analytical expressions and numerical results reported in the main text.

[35] R. F. Harrington, On the Gain and Beamwidth of Directional Antennas, IRE Trans. Antennas Propag. 6, 219 (1958).

[36] R. F. Harrington, Effect of Antenna Size on Gain, Bandwidth, and Efficiency, J. Res. Natl. Bur. Stand. 64D, 1 (1960).

[37] I. Liberal, I. Ederra, R. Gonzalo, and R. W. Ziolkowski, Upper Bounds on Scattering Processes and MetamaterialInspired Structures that Reach Them, IEEE Trans. Antennas Propag. 62, 6344 (2014).

[38] Z. Ruan and S. Fan, Superscattering of Light from Subwavelength Nanostructures, Phys. Rev. Lett. 105, 013901 (2010).

[39] Z. Ruan and S. Fan, Design of Subwavelength Superscattering Nanospheres, Appl. Phys. Lett. 98, 043101 (2011).

[40] R. W. Ziolkowski and A. D. Kipple, Application of Double Negative Materials to Increase the Power Radiated by Electrically Small Antennas, IEEE Trans. Antennas Propag. 51, 2626 (2003).

[41] R. W. Ziolkowski and A. D. Kipple, Reciprocity between the Effects of Resonant Scattering and Enhanced Radiated Power by Electrically Small Antennas in the Presence of Nested Metamaterial Shells, Phys. Rev. E 72, 036602 (2005).

[42] N. Engheta and R. W. Ziolkowski, Metamaterials: Physics and Engineering Explorations (John Wiley \& Sons, New York, 2006).

[43] A. Alù and N. Engheta, How Does Zero Forward-Scattering in Magnetodielectric Nanoparticles Comply with the Optical Theorem?, J. Nanophoton. 4, 041590 (2010).

[44] I. Liberal, I. Ederra, R. Gonzalo, and R. W. Ziolkowski, Induction Theorem Analysis of Resonant Nanoparticles: Design of a Huygens Source Nanoparticle Laser, Phys. Rev. Applied 1, 044002 (2014).

[45] A. Mirzaei, A. E. Miroshnichenko, I. V. Shadrivov, and Y. S. Kivshar, Superscattering of Light Optimized by a Genetic Algorithm, Appl. Phys. Lett. 105, 011109 (2014).

[46] G. T. Di Francia, Super-Gain Antennas and Optical Resolving Power, Nuovo Cimento (1943-1954) 9, 426 (1952).

[47] A. M. H. Wong and G. V. Eleftheriades, SuperdirectivityBased Superoscillatory Waveform Design: A Practical Path to Far-Field Sub-diffraction Imaging, in The 8th European Conference on Antennas and Propagation (EuCAP 2014), The Hague (IEEE, New York, 2014), pp. 1340-1344, http:// ieexplore.ieee.org/document/6902025/citations.

[48] M. L. Morris, M. A. Jensen, and J. W. Wallace, Superdirectivity in MIMO Systems, IEEE Trans. Antennas Propag. 53, 2850 (2005).

[49] N. W. Bikhazi and M. A. Jensen, The Relationship between Antenna Loss and Superdirectivity in MIMO Systems, IEEE Trans. Wireless Commun. 6, 1796 (2007).

[50] T.-I. Lee and Y. E. Wang, A Planar Multipolar Antenna for MIMO Applications, in 2007 IEEE Antennas and Propagation Society International Symposium, Honolulu (IEEE, New York, 2007) pp. 2429-2432, http://ieeexplore.ieee.org/ document/4396023/.

[51] T.-I. Lee and Y. E. Wang, Mode-Based Information Channels in Closely Coupled Dipole Pairs, IEEE Trans. Antennas Propag. 56, 3804 (2008). 
[52] H. A. Wheeler, Fundamental Limitations of Small Antennas, Proc. IRE 35, 1479 (1947).

[53] H. A. Wheeler, The Radiansphere around a Small Antenna, Proc. IRE 47, 1325 (1959).

[54] P. Jin and R.W. Ziolkowski, Metamaterial-Inspired, Electrically Small Huygens Sources, IEEE Antennas Wireless Propag. Lett. 9, 501 (2010).

[55] R. W. Ziolkowski, Low Profile, Broadside Radiating, Electrically Small Huygens Source Antennas, IEEE Access 3, 2644 (2015).

[56] M.-C. Tang, H. Wang, and R. W. Ziolkowski, Design and Testing of Simple, Electrically Small, Low-Profile, Huygens Source Antennas with Broadside Radiation Performance, IEEE Trans. Antennas Propag. 64, 4607 (2016).

[57] M.-C. Tang, T. Shi, and R. W. Ziolkowski, Electrically Small, Broadside Radiating Huygens Source Antenna Augmented with Internal Non-Foster Elements to Increase Its Bandwidth, IEEE Antennas Wireless Propag. Lett. 16, 712 (2016).

[58] B. Q. Wu and K.-M. Luk, A Broadband Dual-Polarized Magneto-Electric Dipole Antenna with Simple Feeds, IEEE Antennas Wireless Propag. Lett. 8, 60 (2008).

[59] K.-M. Luk and B. Wu, The Magnetoelectric Dipole-A Wideband Antenna for Base Stations in Mobile Communications, Proc. IEEE 100, 2297 (2012).

[60] M. Pigeon, C. Delaveaud, L. Rudant, and K. Belmkaddem, Miniature Directive Antennas, Int. J. Microw. Wireless Tech. 6, 45 (2014).

[61] A. Epstein and G. V. Eleftheriades, Passive Lossless Huygens Metasurfaces for Conversion of Arbitrary Source Field to Directive Radiation, IEEE Trans. Antennas Propag. 62, 5680 (2014).

[62] J. P. S. Wong, M. Selvanayagam, and G. V. Eleftheriades, Design of Unit Cells and Demonstration of Methods for Synthesizing Huygens Metasurfaces, Photonic Nanostruct. 12, 360 (2014).

[63] A. Epstein, J. P. S Wong, and G. V. Eleftheriades, CavityExcited Huygens' Metasurface Antennas for Near-Unity Aperture Illumination Efficiency from Arbitrarily Large Apertures, Nat. Commun. 7, 10360 (2016).

[64] A. I. Uzkov, An Approach to the Problem of Optimum Directive Antenna Design, Compt. Rend. Dokl. Acad. Sci. USSR 35, 35 (1946).
[65] E. E. Altshuler, T.H. O’Donnell, A. D. Yaghjian, and S. R. Best, A Monopole Superdirective Array, IEEE Trans. Antennas Propag. 53, 2653 (2005).

[66] S. Lim and H. Ling, Design of a Closely Spaced, Folded Yagi Antenna, IEEE Antennas Wireless Propag. Lett. 5, 302 (2006).

[67] T. H. O’Donnell and A. D. Yaghjian, Electrically Small Superdirective Arrays Using Parasitic Elements, in 2006 IEEE Antennas and Propagation Society International Symposium, Albuquerque (IEEE, New York, 2006), pp. 3111-3114, http://ieeexplore.ieee.org/document/1711268/.

[68] S. Lim and H. Ling, Design of Electrically Small Yagi Antenna, Electron. Lett. 43, 256 (2007).

[69] A. D. Yaghjian, T. H. O’Donnell, E. E. Altshuler, and S. R. Best, Electrically Small Supergain End-Fire Arrays, Radio Sci. 43, 3002 (2008).

[70] A. D. Yaghjian, Increasing the Supergain of Electrically Small Antennas Using Metamaterials, in 2009 3rd European Conference on Antennas and Propagation, Berlin (IEEE, New York, 2009), pp. 858-860, http://ieeexplore .ieee.org/document/5067752/.

[71] S. R. Best, E. E. Altshuler, A. D. Yaghjian, J. M. McGinthy, and T. H. O'Donnell, An Impedance-Matched 2-Element Superdirective Array, IEEE Antennas Wireless Propag. Lett. 7, 302 (2008).

[72] A. Clemente, M. Pigeon, L. Rudant, and C. Delaveaud, Design of a Super Directive Four-Element Compact Antenna Array Using Spherical Wave Expansion, IEEE Trans. Antennas Propag. 63, 4715 (2015).

[73] R. F. Harrington, Time-Harmonic Electromagnetic Fields (McGraw-Hill, New York, 1961).

[74] P.-S. Kildal and S. R. Best, Further Investigations of Fundamental Directivity Limitations of Small Antennas with and without Ground Planes, in 2008 IEEE Antennas and Propagation Society International Symposium, San Diego (IEEE, New York, 2008), pp. 1-4, http://ieeexplore .ieee.org/document/4618968/.

[75] R. O. Thorsen, Diploma thesis, Technical University of Denmark, 2016.

[76] R. W. Ziolkowski, P. Jin, and C.-C. Lin, MetamaterialInspired Engineering of Antennas, Proc. IEEE 99, 1720 (2011).

[77] NewFasant website, https://www.fasant.com/en. 